07.2

\title{
Влияние формы канала на амплитуду случайных телеграфных шумов в подпороговой области беспереходного FinFET-транзистора
}

\author{
(C) М.М. Халиллоев ${ }^{1}$, Б.О. Жаббарова ${ }^{1}$, А.А. Насиров ${ }^{2}$ \\ ${ }^{1}$ Ургенчский государственный университет, Ургенч, Узбекистан \\ ${ }^{2}$ Национальный университет Узбекистана им. М. Улугбека, Ташкент, Узбекистан \\ E-mail: x-mahkam@mail.ru
}

Поступило в Редакцию 3 сентября 2019г.

В окончательной редакции 3 сентября 2019 г.

Принято к публикации 17 сентября 2019 г.

\begin{abstract}
Моделируется зависимость амплитуды случайного телеграфного шума от прироста напряжения на затворе над пороговым для беспереходного вертикального полевого транзистора, изготовленного по технологии „кремний на изоляторе“, с поперечным сечением канала в форме прямоугольника и трапеции. Показано, что в подпороговой области амплитуда шума для транзистора с трапецеидальным поперечным сечением заметно меньше, чем для транзистора с прямоугольным сечением. Помимо этого при одинаковых условиях амплитуда шума при пороговом напряжении на затворе существенно меньше в беспереходном вертикальном транзисторе, чем в планарном, полностью обедненном вертикальном и обычном вертикальном полевом транзисторах.
\end{abstract}

Ключевые слова: случайный телеграфный шум, беспереходный FinFET-транзистор, граничный ловушечный заряд, плотность тока стока.

DOI: 10.21883/PJTF.2019.24.48799.18024

Одним из эффективных путей достижения ультранизкой потребляемой мощности является использование наноразмерных транзисторов металл-оксид-полупроводник (МОП) в подпороговой логике [1]. Малая мощность в этом случае достигается за счет использования в схемах подпороговой логики нанометровых МОП-транзисторов, работающих в подпороговом или околопороговом режиме с рабочими токами наноамперного диапазона. При масштабировании МОП-транзисторов возникают различные деградационные эффекты. Среди них можно отметить короткоканальные эффекты [2] и усиление чувствительности различных характеристик к встраиванию локального заряда в диэлектрическом слое $[3,4]$ или единичного заряда в подзатворном диэлектрическом слое либо на границе подзатворного диэлектрика с каналом, приводящего к проявлению случайных телеграфных шумов (СТШ) в токе стока [5]. Для ослабления короткоканальных эффектов, возникающих в МОП-транзисторах при уменьшении их размеров до нанометровых масштабов, разработаны трехзатворные вертикальные полевые транзисторы (FinFET-транзисторы). Вслед за FinFETтранзисторами сравнительно недавно в качестве альтернативы для приборов с длинами канала менее $20 \mathrm{~nm}$ предложены беспереходные (JL) FinFET-транзисторы [2], технология изготовления которых существенно проще и дешевле, так как исключает процесс формирования стоковых и истоковых областей и позволяет избежать соответствующих проблем, связанных с легированием этих наноразмерных областей. JL FinFET-транзисторы представляют собой сильнолегированный (обычно в пределах $10^{18}-10^{19} \mathrm{~cm}^{-3}$ ) однородный тонкий полупро- водниковый резистор с изолированным затвором, который управляет потоком носителей заряда в резисторе между стоком и истоком. В отличие от традиционных МОП-транзисторов, работающих в режиме инверсии, JL FinFET-транзисторы работают в режиме аккумуляции.

Короткоканальные эффекты в JL FinFET-транзисторах изучаются довольно широко [6-8], в то время как исследования влияния единичных оксидных или граничных ловушечных зарядов, вызывающих СТШ-сигналы в этих транзисторах, на ток стока, а также работы, связанные с применением этих транзисторов в логике ультранизкой мощности, находятся на начальной стадии. Есть работы, в которых основное внимание уделяется зависимости амплитуды СТШ-сигнала от технологии изготовления транзистора, температуры, напряжения на затворе и стоке в режиме аккумуляции $[9,10]$, зависимости показателей эффективности работы беспереходного транзистора в логике ультранизкой мощности от уровня легирования канала, диэлектрической постоянной материала спейсера, сопротивления канала [11]. Однако имеется очень мало работ, в которых исследуется влияние технологических флуктуаций геометрических размеров и отклонений от предполагаемых форм при изготовлении наноразмерных JL FinFET-транзисторов на их шумовые характеристики. В то же время технологические флуктуации геометрических размеров могут приводить к заметному изменению ожидаемых параметров и характеристик транзистора. Так, в работах [12-14] показана зависимость изменения характеристик, параметров и короткоканальных эффектов от изменения формы канала и размеров FinFET-транзистора, работающего в инверсной моде. 

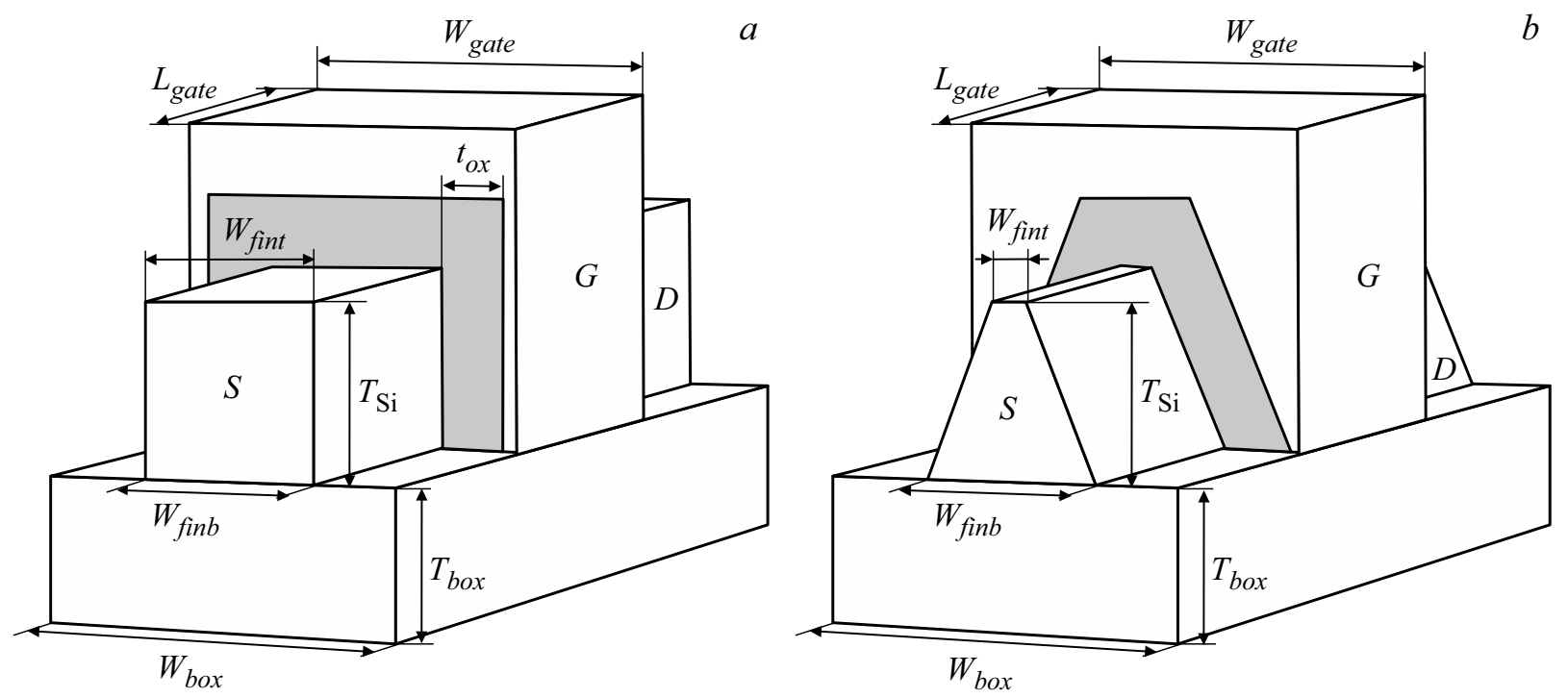

Рис. 1. Моделируемая структура беспереходного FinFET-транзистора с прямоугольным $(a)$ и трапецеидальным (b) поперечным сечением канала.

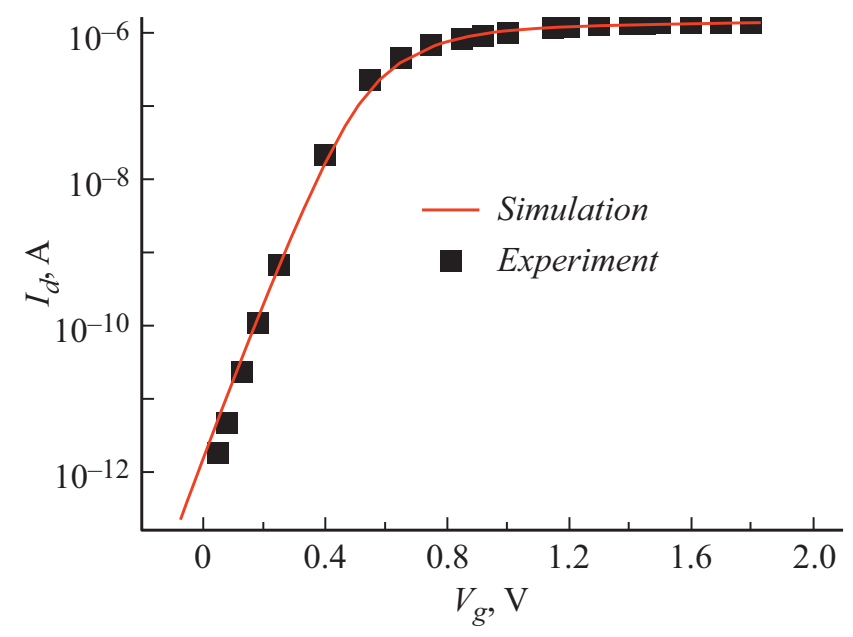

Рис. 2. Зависимость тока стока от напряжения на затворе моделируемого транзистора (линия) и экспериментального образца (точки) с подобными размерами и параметрами.

В настоящей работе моделируется зависимость амплитуды СТШ-сигнала, индуцированного единичным зарядом, захваченным на граничном дефекте в центре потолка канала JL FinFET-транзистора с различной формой канала, изготовленного на основе технологии „кремний на изоляторе“ (КНИ), структура которого представлена на рис. 1. Рассматривались каналы в форме усеченной треугольной призмы и параллелепипеда, которые в поперечном сечении проявляются в виде прямоугольника и трапеции (рис. $1, a, b)$.

3D-моделирование проводилось с использованием программы Advanced TCAD Sentaurus [15]. При моделировании использовалась диффузионно-дрейфовая модель. В модели учитывались зависимость подвижности от концентрации легирования и насыщение скорости носителей, а также влияние нормальной составляющей поля на ток стока. Поскольку размеры транзистора имеют нанометровые масштабы, необходимо принимать во внимание и квантовые эффекты. Наиболее предпочтительной при диффузионно-дрейфовом моделировании является квантовая корректировка по градиенту плотности $[16,17]$, которая использовалась в настоящей работе. Используемая модель калибровалась по экспериментальным результатам работы [18] (рис. 2). При моделировании использовались следующие параметры структуры. Длина TiN-затвора $(G)$ равна $L_{\text {gate }}=30 \mathrm{~nm}$.

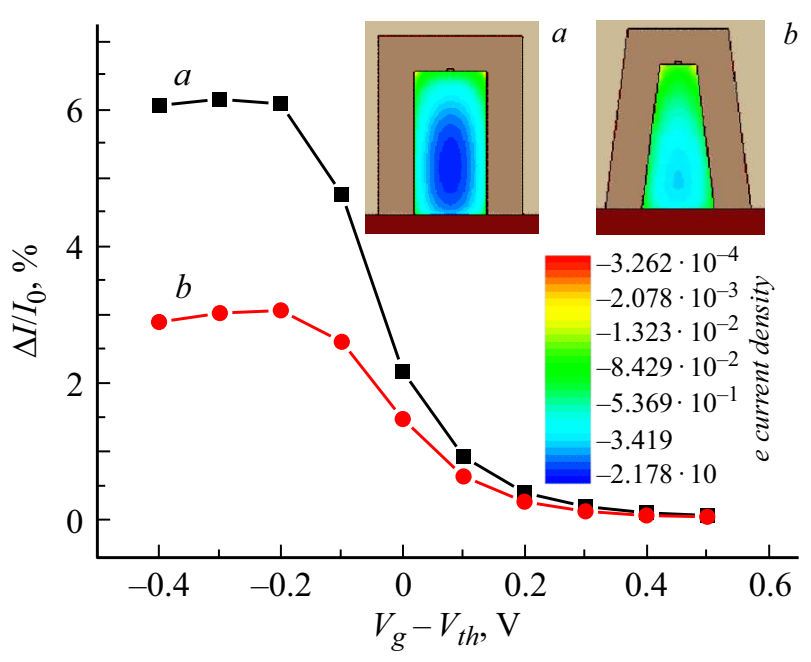

Рис. 3. Зависимость амплитуды СТШ-сигнала от прироста напряжения на затворе для моделируемых беспереходных FinFET-транзисторов с различной формой канала. На вставках показано распределение плотности электронного тока в поперечном сечении в центре канала для транзистора с прямоугольным $(a)$ и трапецеидальным $(b)$ сечением. 
Максимальная амплитуда СТШ-сигнала для МОП-транзисторов, соответствующих различным технологиям

\begin{tabular}{|c|c|c|c|c|c|c|c|c|c|}
\hline \multirow{2}{*}{$\begin{array}{l}\text { № } \\
\text { п/п }\end{array}$} & \multirow[b]{2}{*}{ Технология } & \multicolumn{6}{|c|}{ Геометрические размеры } & \multirow{2}{*}{$\begin{array}{c}\text { Лит. } \\
\text { ссылка }\end{array}$} & \multirow{2}{*}{$\begin{array}{c}\text { Максимальная } \\
\text { амплитуда } \\
\text { СТШ-сигнала } \\
\text { при } V_{g}=V_{t h}, \%\end{array}$} \\
\hline & & $\begin{array}{l}L_{g}, \\
\mathrm{~nm}\end{array}$ & $\begin{array}{l}t_{\text {eff }}, \\
\mathrm{nm}\end{array}$ & $\begin{array}{c}T_{b o x} \\
\mathrm{~nm}\end{array}$ & $\begin{array}{c}W_{b o x} \\
\mathrm{~nm}\end{array}$ & $\begin{array}{c}T_{\mathrm{Si}} \\
\mathrm{nm}\end{array}$ & $\begin{array}{l}W_{\mathrm{Si}} \\
\mathrm{nm}\end{array}$ & & \\
\hline 1 & Bulk MOSFET & 22 & 0.9 & & & & 22 & [19] & 28 \\
\hline 2 & FDSOI & 22 & 0.9 & 10 & 10 & 6 & 10 & [19] & 26 \\
\hline 3 & FinFET & 22 & 0.9 & 25 & 10 & 20 & $<10 \mathrm{~nm}$ & {$[19]$} & $8-22$ \\
\hline 4 & JL FinFET & 30 & 0.9 & 25 & 48 & 20 & $\begin{array}{c}W_{\text {fint }}=10 \\
W_{\text {fint }}=5\end{array}$ & $\begin{array}{l}\text { Наст. раб. } \\
\text { Наст. раб. }\end{array}$ & $\begin{array}{c}2 \\
1.5\end{array}$ \\
\hline
\end{tabular}

В качестве подзатворного оксидного слоя взят $\mathrm{HfO}_{2}$, эквивалентная толщина составляла $t_{e f f}=0.9 \mathrm{~nm}$. Ширина и толщина скрытого оксидного слоя равны соответственно $W_{b o x}=48 \mathrm{~nm}$ и $T_{b o x}=25 \mathrm{~nm}$. Параметром, отражающим форму канала, является ширина его потолка $W_{f i n t}$, которая для усеченной треугольной призмы равна $5 \mathrm{~nm}$. Канал транзистора легирован бором с концентрацией $5 \cdot 10^{18} \mathrm{~cm}^{-3}$ и имеет ширину у основания $W_{f i n b}=10 \mathrm{~nm}$.

Результаты моделирования зависимости амплитуды СТШ-сигнала от прироста напряжения на затворе $V_{g}$ от порогового $V_{t h}\left(V_{g}-V_{t h}\right)$ для двух форм канала представлены на рис. 3. Из результатов видно, что при пороговых и меньших напряжениях на затворе амплитуда СТШ-сигнала заметно увеличивается по сравнению с надпороговым значением. При этом при прямоугольном сечении канала максимальная амплитуда шума в подпороговых областях почти в 2 раза больше, чем для случая с трапецеидальным сечением. Это связано с тем, что расстояние от единичного граничного ловушечного заряда, расположенного в середине потолка канала, до сечения с максимальным током стока меньше для канала с прямоугольным сечением (вставка $a$ на рис. 3 ), чем для канала с трапецеидальным сечением (вставка $b$ на рис. 3). Наблюдаемые амплитуды СТШ в околопороговой области $\left(V_{g}=V_{t h}\right)$ для обеих форм канала JL FinFET-транзистора имеют заметно меньшие значения, чем для планарного МОП-транзистора на объемных кристаллах, транзистора с полностью обедненным каналом (FDSOI) и переходного FinFET-транзистора с примерно одинаковыми геометрическими параметрами, значения которых взяты из работы [19] (см. таблицу). Даже в подпороговых областях $\left(V_{g}<V_{t h}\right)$ величина амплитуды СТШ-сигнала в КНИ JL FinFET-транзисторах меньше, чем амплитуда в указанных выше приборах, соответствующая пороговому напряжению $\left(V_{g}=V_{t h}\right)$. В таблице для всех случаев приведены амплитуды СТШсигнала, индуцированного единичным граничным ловушечным зарядом, локализованным в середине потолка канала.

Таким образом, результаты моделирования показывают, что в КНИ JL FinFET-транзисторе амплитуда СТШ-сигнала зависит от формы канала: она меньше для транзистора с каналом трапецеидального поперечного сечения, чем в случае канала прямоугольного сечения. При пороговом напряжении в КHИ JL FinFET-транзисторах проявляется меньшая амплитуда СТШ-сигнала, чем в планарном MOП-, FDSOI-, FinFET-транзисторах примерно одинаковых геометрических размеров. Это свойство указывает на преимущества использования КНИ JL FinFET-транзисторов с трапецеидальным сечением в подпороговой логике с ультранизкой мощностью.

\section{Конфоликт интересов}

Авторы заявляют, что у них нет конфликта интересов.

\section{Список литературы}

[1] Marcovic D., Cheng C.W., Alarcon L.P., Liu T.-T, Rabaey J.M. // Proc. of the IEEE. 2010. V. 98. N 2. P. 237252.

[2] Ferain I., Colinge C.A., Colinge J.-P. // Nature. 2011. V. 479. N 7373. P. $310-316$.

[3] Атамуратова 3.А., Юсупов А., Халикбердиев Б.О., Атамуратов А.Э. // ЖТФ. 2019. Т. 89. В. 7. С. 1067-1070.

[4] Atamuratov A.E., Atamuratova Z.A., Yusupov A., Ghani A. // Results Phys. 2018. V. 11. P. 656-658.

[5] Campbell J.P., Yul L.C., Cheung K.P., Qin J., Suehle J.S., Oates A., Sheng $K$. Large random telegraph noise in subthreshold operation of nano-scale nMOSFETs // Proc. IEEE Int. Conf. IC Design and Technology (ICICDT). IEEE, 2009. P. 17-20.

[6] Paz B.C., Ávila-Herrera F., Cerdeira A., Pavanello M.A. // Semicond. Sci. Technol. 2015. V. 30. N 5. P. 055011.

[7] Jaiswal N., Kranti A. // IEEE Trans. Electron Dev. 2018. V. 65. N 9. P. 3669-3675.

[8] Razavi P., Dehdashti-Akhavan $N ., \quad Y u$ R., Fagas G., Ferain I., Colinge J.-P. Investigation of short-channel effects in junctionless nanowire transistors // Extended Abstracts of the 2011 Int. Conf. on solid state devices and materials. Nagoya, 2011. P. 106-107.

[9] Fan M.-L., Yang S.-Y., Hu V.P.-H., Chen Y.-N., Su P., Chuang C.-T. // Microelectron. Reliability. 2014. V. 54. N 4. P. 698-711.

[10] Nazarov A.N., Ferain I., Akhavan N.D., Razavi P., Yu R., Colinge J.P. // Appl. Phys. Lett. 2011. V. 98. N 9. P. 092111.

[11] Roy D., Biswas A. // Superlatt. Microstruct. 2016. V. 97. P. $140-154$. 
[12] Абдикаримов А.Э., Юсупов А., Атамуратов А.Э. // Письма в ЖТФ. 2018. Т. 44. В. 21. С. 22-29.

[13] Atamuratov A.E., Abdikarimov A.E., Khalilloev M.M., Atamuratova Z.A., Rahmanov R., Garcia-Loureiro A., Yusupov A. // Nanosystems: Phys. Chem. Math. 2017. V. 8. N 1. P. 71-74.

[14] Abdikarimov A.E., Indalecio A.G., Comesana E., Seoane N., Kalna K., Garcia-Loureiro A.J., Atamuratov A.E. Influence of device geometry on electrical characteristics of a $10.7 \mathrm{~nm}$ SOI-FinFET // 17th Int. Workshop on computational electronics. Paris, 2014. P. 247-248.

http://www.iwce.org/iwce/browse/ iwce-17/ abstracts. html

[15] http://www.synopsys.com

[16] García-Loureiro A.J., Seoane N., Aldegunde M., Valín R., Asenov A., Martinez A., Kalna K. // IEEE Trans. ComputerAided Design Integr. Circuits Syst. 2011. V. 30. N 6. P. 841851.

[17] Asenov A., Brown A., Watling J. // Solid-State Electron. 2003. V. 47. N 7. P. $1141-1145$.

[18] Barraud S., Berthomé M., Coquand R., Cassé M., Ernst T., Samson M.-P., Perreau P., Bourdelle K.K., Faynot O., Poiroux T. // IEEE Electron Dev. Lett. 2012. V. 33. N 9. P. 1225-1227.

[19] Gerrer L., Amoroso S.M., Hussin R., Asenov A. // Microelectron. Reliability. 2014. V. 54. N 9-10. P. 1749-1752. 